\title{
Editorials
}

\section{Social prescribing in general practice}

\section{WHAT IS SOCIAL PRESCRIBING?}

Social prescribing is a health pathway which enables GPs and others to refer patients with psychosocial challenges onto non-clinical support and activities within their local community. This is usually facilitated by a social prescribing link worker who may also offer one-to-one support to the patient in the form of a brief coaching intervention. With the recent commitment set out by The NHS Long Term Plan to recruit 1000 new social prescribing link workers by 2020/2021, link worker social prescribing has now come of age as a mainstream health intervention. While there has been controversy around the evidence base; ${ }^{2}$ as a 'common sense' approach to supporting those whose health challenges have a psycho-social component, social prescribing has always had much vocal support. There are now numerous social prescribing initiatives located within primary care services across the UK and a wealth of evaluations have documented not just patient health outcomes, but also the challenges of setting up such a service. ${ }^{3}$ This editorial will look at some of these challenges, as well as identify other important factors to take into consideration when designing and delivering a social prescribing intervention within primary care.

There are various different models of social prescribing ${ }^{4}$ encompassing a range of interventions from straight signposting, where patients are directed to the nearest support group or activity within their local community, through to what can be described as a brief coaching intervention where patients receive more intensive 'holistic' support from a link worker. Link worker social prescribing has been shown to be particularly effective for those patients who need help with behaviour change and overcoming barriers to accessing further support elsewhere. ${ }^{5}$ However, this model of social prescribing inevitably comes at greater financial cost as link workers need to not only be employed, but trained and supported in a way that allows them to conduct their work safely and effectively. ${ }^{6}$

\section{SETTING UP A SOCIAL PRESCRIBING PATHWAY}

One of the first things to take into consideration when setting up a social prescribing service is identifying who can be realistically helped with such an intervention. There can be a temptation to refer on any

\section{"Link worker social prescribing has been shown to be particularly effective for those patients who need help with behaviour change and overcoming barriers to accessing further support elsewhere.}

patient who is challenging to treat through the usual clinical pathways, which can lead to the social prescribing service becoming overwhelmed, impacting negatively on both those referred inappropriately, as well as directing resources away from those who could most benefit. Understanding the needs of the local population is key. For example, if there are large numbers of socially isolated older people in the area are there the resources to help them within the community? It is important to undertake asset mapping as part of the design process in order to be confident that the necessary support or activities are available. This, in itself, can be a challenge as voluntary services come and go with funding cycles, and obtaining an up-to-date picture of what is available at any given time can be a huge task.

While there is often an enormous variety of support available in densely populated areas such as London, databases are often not kept up-to-date, and for link workers, building and maintaining relationships with organisations within their local community is a time-consuming but necessary part of their role which is not always acknowledged within their workload. ${ }^{7}$ Highly specialist support is often the hardest to find and the first to have its funding cut. ${ }^{8}$ Patients managing long-term conditions and comorbidities may require more specific support than those experiencing low level mental health or social isolation, where a variety of activities can be enough to expand the patient's social connections and ultimately help to improve their wellbeing. Sometimes, it is not immediately obvious what kind of support is required and issues only come to light later in the process rather than at referral. For example, homelessness is a common challenge in London where social prescribing schemes can do little to alleviate the problem. ${ }^{8}$

The means of referral will also need to be decided. It may be desirable to refer patients via, not only their GP, but also adult social care services or even through self-referral. However, if there is not the capacity in the system, there is again a danger of overloading both link workers and community or statutory organisations, which could potentially divert scarce resources from the intended target group. One way of managing high numbers of referrals might be to set up a triage system where a patient's needs can be initially assessed before either offering them straight signposting or, if needed, a number of more intensive one-to-one sessions with a link worker. While it is generally agreed that the link worker role should not be a clinical one, in order to facilitate a more collaborative peer relationship, it is often practical to base link workers in GP surgeries, but this does not necessarily have to be the case and many schemes locate them within the community.

Link workers themselves can operate in a variety of ways. They may meet the patient face-to-face and sometimes even accompany them to activities or support groups. The greatest advantage of this method of delivery is that patients have the opportunity to build a 'trust' relationship with their link worker, which may then serve as a model for building similar relationships elsewhere. This can have a powerful impact on somebody who has never experienced such relationships in their life before. Less intensive methods of delivery might involve link workers offering a telephone intervention, or even access online. While this is a less costly way of providing the service, it is unlikely to have the same level of impact as a face-to-face intervention, although for some patients the anonymity it affords may make it easier for them to engage when a face-to-face session might feel too confrontational. ${ }^{8}$ However, when there is any contact with patients, no matter 


\section{"One of the first things to take into consideration when setting up a social prescribing service is identifying who can be realistically helped with such an intervention.}

\section{ADDRESS FOR CORRESPONDENCE}

\section{Caroline Frostick}

Institute for Health and Human Development University of East London, Water Lane, Stratford, London E15 4LZ, UK.

Email: c.frostick@uel.ac.uk how tight the referral criteria might be, there is always the possibility that a patient may present with a serious safeguarding issue that cannot be ignored. It is therefore essential that all link workers are not only trained to deal with this eventuality, but are also provided with proper support in the form of clinical supervision, where they can discuss challenging patients and situations. ${ }^{6}$

It is important for the sustainability of the service that feedback mechanisms are developed and maintained with all parties concerned. GPs need to know outcomes for patients or they will stop referring, and community organisations in particular can feel taken advantage of if they are not formally involved in the service. ${ }^{7}$ An ongoing and justified complaint from community sector organisations existing on shoe-string budgets is that social prescribing pathways often increase their workload with no extra funding made available, although there are exceptions to this. ${ }^{8}$ Co-production of social prescribing schemes with service users should also be encouraged, not just as a 'consultation', but as an appropriate co-production, particularly at the design stage, but ideally throughout the implementation and evaluation stages.?

A final important consideration in setting up a social prescribing pathway within primary care is the need to evaluate the service's effectiveness. The use of measurement tools needs careful consideration as traditional clinical measures may not capture meaningful change in the patient. A mixed methods approach is advisable for this reason, as is the use of tools which give primacy to the patient's perspective; for example, 'Measure Yourself Concerns and Wellbeing'. ${ }^{10}$ Social prescribing is a complex health intervention and significant change for the patient can occur not just at the end of the pathway, in the form of community support and activities, but often at the link worker stage, where a trust relationship and a coaching intervention can be the focal point of sustainable behaviour change and other forms of support. ${ }^{3}$ Another unresolved problem in assessing the effectiveness of social prescribing is that current evaluations usually do not follow what happens to service users after they have left the link worker, the types of activities they engage in, and how often. There is growing need for more robust evaluations involving larger sample sizes, multisites, longer-term follow-ups, and ideally a control group to more effectively compare health outcomes.

\section{CONCLUSION}

At its best, social prescribing has the potential to relieve pressure on GPs and primary care services by offering an alternative pathway to patients whose needs are unable to be addressed through the usual medical routes. Usually, it is part of the link worker's role to set the service up, making contact and sustaining relationships with health professionals and community and statutory services. As such, there is usually little extra work involved from the GP's perspective, and in fact the major challenge for GPs tends to be remembering to refer patients on to the service. Once it is up and running, the social prescribing can then provide an effective 'bridge' between the medical expertise in the health service and contextual social expertise in the community.

\section{Caroline Frostick,}

Research Fellow, University of East London, Institute for Health and Human Development, Stratford, London, UK.

\section{Marcello Bertotti,}

Senior Research Fellow, University of East London, Institute for Health and Human Development, Stratford, London, UK.

\section{Provenance}

Commissioned; externally peer reviewed.

\section{Competing interests}

The authors have declared no competing interests.

DOI: https://doi.org/10.3399/bjgp19X706157

\section{REFERENCES}

1. NHS England. The NHS Long Term Plan. 2019. https://www.longtermplan.nhs.uk/wp-content/ uploads/2019/08/nhs-long-term-plan-version1.2.pdf laccessed 8 Oct 2019).

2. Bickerdike L, Booth A, Wilson PM, et al. Social prescribing: less rhetoric and more reality. A systematic review of the evidence. BMJ Open 2017; 7(4): e013384. DOI: 10.1136/ bmjopen-2016-013384.

3. Bertotti $M$, Frostick $C$, Hutt $P$, et al. A realist evaluation of social prescribing: an exploration into the context and mechanisms underpinning a pathway linking primary care with the voluntary sector. Prim Health Care Res Dev2018; 19(3): 232-245. DOI: 10.1017/S1463423617000706.

4. Kimberlee R. What is social prescribing? Adv Soc SciRes J2015; 2(1): 102-110. DOI: 10.14738/ assrj.21.808

5. Moffatt S, Steer M, Lawson S, et al. Link Worker social prescribing to improve health and wellbeing for people with long-term conditions: qualitative study of service user perceptions. BMJ Open 2017; 7(7): e015203. DOI: 10.1136/ bmjopen-2016-015203

6. Frostick C, Bertotti, M The frontline of social prescribing - how do we ensure Link Workers can work safely and effectively within primary care? Chronic Illness 2019 (in press).

7. City and Hackney Clinical Commissioning Group, University of East London. Shine 2014 final report. Social Prescribing: integrating GP and community assets for health. 2015. https:// mww.health.org.uk/sites/default/files/City\%20 and\%20Hackney\%20CCG\%20final\%2Oreport.pdf laccessed 8 Oct 2019).

8. Bertotti M, Frostick C, Tong J, Netuveli G. The social prescribing service in the London Borough of Waltham Forest: final evaluation report. 2017. https://uww.researchgate.net/ publication/320280866_The_Social_Prescribing service_in_the_London_Borough_of_Waltham Forest_final_evaluation_report laccessed 20 Sep 2019).

9. Dayson C. Social prescribing 'plus': a model of asset-based collaborative innovation? People, Place and Policy 2017; 11(2): 90-104. DOI: 10.3351/ppp.2017.4839587343

10. Paterson C, Thomas K, Manasse A, et al. Measure Yourself Concerns and Wellbeing (MYCaW): an individualised questionnaire for evaluating outcome in cancer support care that includes complementary therapies. Complement THER Med 2007; 15(1): 38-45. 